\title{
EDITORIAL
}

\section{Improving Weight Management among Veterans}

\author{
David Atkins, MD, MPH \\ VA Health Services Research and Development Service, Washington, DC, USA.
}

J Gen Intern Med 32(Suppl 1):S1-S3

DOI: $10.1007 / \mathrm{s} 11606-017-4007-0$

(C) Society of General Internal Medicine 2017

$\mathrm{T}$ he steady increase in the numbers of patients who are overweight or obese is a serious challenge to healthcare systems, ${ }^{1}$ including the Veterans Health Administration (VHA). Obesity is an important driver of increasing morbidity related to hypertension, diabetes, certain cancers, and osteoarthritis. It is also an important driver of healthcare costs as Veterans and non-Veterans age. In a recent study of nearly 5 million VA primary care patients $(347,112$ females and $4,567,096$ males), $37 \%$ were overweight and $41 \%$ were obese. $^{2}$

Especially concerning is the steep rise in the number of individuals in the USA with "super obesity," defined as a body mass index (BMI) of $\geq 50 \mathrm{~kg} / \mathrm{m}^{2}$, a population that grew more rapidly between 1986 and 2010 than the population who were overweight (BMI 25-29.9) or obese (BMI $\geq 30$ ). Individuals with BMI $\geq 50$ not only have more complex health issues, but also have higher risk for post-surgical complications after weight loss surgery. ${ }^{3}$

Like many large health systems trying to address the obesity epidemic, the VA healthcare system offers three primary weight management interventions: (1) the MOVE! Weight Management Program, a system-wide behavior counseling program aimed at increasing physical activity and promoting healthy eating, (2) weight loss medications, and (3) bariatric surgery. From 2014 to 2015, approximately 263,000 Veterans participated in MOVE!; from 2012 to 2015, an average of about 2500 Veterans obtained weight loss medications annually; and between 2010 and 2014, approximately 1,700 Veterans underwent bariatric surgery procedures. ${ }^{4}$ The availability of weight management interventions across VA medical centers and coordination of interventions within VA medical centers likely vary.

A unique and pressing opportunity in the delivery of weight management interventions in VA (and many US and international health systems) is the adoption of a population-based approach to ensure that the $65-70 \%$ of patients who are eligible for weight loss interventions are matched with the right treatment based on their needs. In 2016, VA's Health Services Research and Development (HSR\&D) Service held a

Published online February 21, 2017 state-of-the-art conference (SOTA) entitled "Weight Management in the Veterans Health Administration." The overall goal of the conference was to help describe a more integrated system-wide approach to improving weight management for Veterans and identify research priorities in this domain. This Journal of General Internal Medicine (JGIM) Supplement, funded by HSR\&D, includes findings from the conference and summaries from the three workgroups that began by independently considering behavioral interventions, pharmacotherapy, and bariatric surgery. Unique to this SOTA (as reported in the paper by Raffa) was a discussion among the combined workgroups about how to integrate all three approaches. This supplement also includes original papers selected from manuscripts submitted in response to a Call for Papers following the conference.

\section{BEHAVIORAL INTERVENTIONS}

Masheb and colleagues' paper summarizes discussion, policy recommendations, and research recommendations of the Behavioral Interventions Workgroup. Their top policy recommendations were to establish a system-wide culture for weight management and to identify a population-level health metric to measure the impact of weight management interventions. Such a metric could then be tracked and clearly communicated throughout the VA healthcare system to show whether we are making progress. The Workgroup's top research recommendation was to determine how to deploy and scale the most effective behavioral weight management interventions for Veterans across the diverse medical facilities (168 medical centers and over 1000 community clinics) that make up the VHA. ${ }^{5}$

Compared to overweight and obese non-Veterans, obese or overweight patients in VHA are more likely to have underlying mental health problems that may complicate efforts to achieve clinically meaningful weight loss. In a paper examining weight management designed for these patients, Erickson and colleagues investigated behavioral weight management interventions for Veterans with mental illness across four VA medical centers. One group of Veterans received "Lifestyle Balance" (LB) consisting of classes and individual nutritional counseling with a dietitian, modified from the Diabetes Prevention Program. A second group of Veterans received less intensive "Usual Care" (UC) consisting of weight monitoring and provision of self-help materials. Participants in both the LB and UC groups lost weight; however, LB participants also demonstrated important changes in nutritional behaviors by 
reducing overall caloric intake and improving caloric quality by decreasing consumption of empty calories. In addition, these Veterans decreased their waist circumference and adiposity, reflecting changes in body composition from exercise and weight loss. Changes in intermediate measures (e.g., nutritional behaviors, waist circumference, and adiposity) suggested possible long-term reductions in the risk from cardiovascular and metabolic diseases ${ }^{6}$ if these improvements can be sustained over the long term.

\section{PHARMACOTHERAPIES}

The paper by Semla and colleagues summarizes recommendations from the Pharmacotherapy Workgroup. Faced with the low rate of pharmacotherapy prescribing in VHA $(<2 \%$ of all patients in MOVE!), the Workgroup examined medication effectiveness, which patients benefit, and what barriers to appropriate prescribing exist in VHA. Barriers included patient and provider concerns about medication safety and efficacy and limited involvement of primary care in pharmacotherapy. These might be addressable via patient and provider education about weight management medication, the health benefits of weight loss, and the creation of a system to help navigate patients through weight management treatment options. In response to the Workgroup recommendations, VHA Pharmacy Benefits Management has revised its Criteria for Use of weight management medications to increase their availability. Among the research recommendations were studies examining a broader set of long-term outcomes (beyond 2 years) after medication therapy and studies addressing the timing and duration of drug therapy. ${ }^{7}$

\section{BARIATRIC SURGERY}

Peterson and colleagues conducted a rapid evidence review to help better understand bariatric surgery outcomes in adults with a $B M I \geq 50 \mathrm{~kg} / \mathrm{m}^{2}$. Their findings showed limited evidence that bariatric surgery is associated with increased mortality in the first year for adults with super obesity compared to usual care, but decreased longer-term mortality. Despite the increased surgical risk, existing evidence suggests that people with a BMI $\geq 50$ may benefit from bariatric surgery. ${ }^{3}$ This review also identified many evidence gaps about the benefits of bariatric surgery for adults with a $B M I \geq 50 \mathrm{~kg} / \mathrm{m}^{2}$.

Funk and colleagues summarize recommendations for future research from the Bariatric Surgery Workgroup. They identified and prioritized several research gaps including: developing effective pre- and postoperative interventions, documenting long-term bariatric surgery outcomes, and establishing a registry of obese Veterans. They also suggest that a comprehensive workforce assessment would help clarify VHA's long-term capacity to increase access to weight management resources. A better understanding of the patient, provider, and health system barriers and facilitators to bariatric surgery care was seen as critical to increasing the appropriate use of surgery in the care of overweight or obese Veterans. ${ }^{8}$

\section{CREATING INTEGRATED WEIGHT MANAGEMENT SOLUTIONS}

The increasing prevalence of Veterans who are overweight and obese suggests that high-quality weight management will need to become as ubiquitous as primary care and will likely require effective and strategic coordination across interventions, especially in large health systems. This is a management and service delivery challenge that does not yet appear to have been solved in health systems in the US or abroad. To stimulate discussion about how to address a clinical challenge of this magnitude, Raffa and colleagues summarize the themes from all three workgroups regarding challenges in developing an integrated population-based approach that incorporates behavioral, medication, and surgical treatments. Their paper notes four overlapping tasks: (1) implementing an evidencebased multicomponent approach to weight management; (2) providing access to overweight and obesity care across VHA; (3) promoting care coordination and patient-engagement strategies; (4) stratifying treatment by the level of patient risk. ${ }^{9}$ An accompanying editorial by Pronk discusses the potential role of systems science, which represents a broad approach that recognizes the importance of the whole - and the interdependence of the component parts that contribute to obesity. These include an individual's past experiences, behaviors, family dynamics, workplace culture, community design, and environmental exposures. ${ }^{4}$

Additional articles in this Supplement focus on several other important issues related to weight management. Young and colleagues discuss the benefits of using technology-delivered weight management with peer coaching to overweight Veterans who have serious mental illness. ${ }^{10}$ Lutes and colleagues examine the effectiveness of a second year of low intensity support for small changes in weight loss compared to the usual care approach used in MOVE! Programs. ${ }^{11}$ Chan and colleague examine the relationship between Veterans participating in the MOVE! Program and weight outcomes. ${ }^{12}$ Breland and colleagues examine the prevalence of obesity among key populations of male and female Veterans, including racial/ ethnic and comorbidity sub-populations, ${ }^{2}$ while Estabrooks and colleagues assess the impact of a community-based weight loss program among non-Veterans. ${ }^{13}$

VA HSR\&D believes this Supplement will make a significant contribution to establishing an integrated approach to weight management in the VHA, one that will better help Veterans obtain effective, patient-centered interventions to assist them in reaching a healthier weight. As representatives of the nation's largest integrated healthcare system, serving more than 6 million patients each year, we are optimistic about creating a more integrated, population-based approach to 
obesity that will improve the health of Veterans and offer other healthcare systems an effective model for addressing the rising tide of obesity facing our country today.

\section{Acknowledgements:}

Contributor: The author would like to thank Ms. Diane Hanks for her help with this manuscript. Ms. Hanks is part of HSR\&D's Center for Information Dissemination and Education Resources in Boston, MA.

Corresponding Author: David Atkins, MD, MPH; VA Health Services Research and Development Service, 810 Vermont Ave., NW (10P9662), Washington, DC 20420, USA (e-mail: David.Atkins@va.gov).

\section{Compliance with Ethical Standards:}

Conflict of Interest: The author is a Federal employee and reports no conflicts of interests regarding this article. The opinions are those of the author and do not refelct official policy of the Department of Veterans Affairs.

\section{REFERENCES}

1. Flegal KM, Kruszon-Moran D, Carroll MD, et al. Trends in obesity among adults in the United States, 2005 to 2014. JAMA. 2016;315(21):2284-2291

2. Breland JY, Phibbs CS, Hoggatt KJ, et al. The obesity epidemic in the Veterans Health Administration: Prevalence among key populations of women and men Veterans. J Gen Intern Med. 2016. doi:10.1007/ s11606-016-3962-1.

3. Peterson K, Anderson J, Boundy E, et al. Rapid evidence review of bariatric surgery in super obesity. J Gen Intern Med. 2016. doi:10.1007/ s11606-016-3950-5.
4. Pronk NP. A systems science perspective on addressing obesity at the Veterans Health Administration. J Gen Intern Med. 2016. doi:10.1007/ s11606-016-3975-9.

5. Masheb RM, Chan SH, Raffa SD, et al. State-of-the-art conference on weight management: Policy and research recommendations for advancing behavioral interventions. J Gen Intern Med. 2016. doi:10.1007/ s11606-016-3965-y.

6. Erickson ZD, Kwan CL, Gelberg HA, et al. A randomized, controlled multisite study of behavioral interventions for Veterans with mental illness and antipsychotic medication-associated obesity. J Gen Intern Med. 2016. doi:10.1007/s11606-016-3960-3.

7. Semla TP, Ruser C, Good CB, et al. Pharmacotherapy for weight management in VHA. J Gen Intern Med. 2016. doi:10.1007/s11606016-3949-y.

8. Funk LM, Gunnar W, Dominitz JA, et al. A health services research agenda for bariatric surgery within the Veterans Health Administration. J Gen Intern Med. 2016. doi:10.1007/s11606-016-3951-4.

9. Raffa SD, Maciejewski ML, Zimmerman LE, et al. A system-level approach to overweight and obesity in the Veterans Health Administration. J Gen Intern Med. 2016. doi:10.1007/s11606-016-3948-z.

10. Young AS, Cohen AN, Goldberg $\mathbf{R}$, et al. Improving weight in people with serious mental illness: The effectiveness of computerized services with peer coaches. J Gen Intern Med. 2016. doi:10. 1007/s11606-016-3963-0.

11. Lutes LD, Damschroder LJ, et al. Behavioral treatment for Veterans with obesity: 24-month weight outcomes from the ASPIRE-VA Small Changes randomized trial. J Gen Intern Med. 2017. doi:10.1007/s11606-017-3987-0.

12. Chan SH, Raffa SD. Examining the dose-response relationship in the Veterans Health Administration's MOVE! Weight management program: a nationwide observational study. J Gen Intern Med. 2017. doi:10.1007/ s11606-017-3992-3.

13. Estabrooks PA, Wilson KE, McGuire TJ, et al. A quasi-experiment to assess the impact of a scalable, community-based weight loss program: Combining reach, effectiveness, and cost. J Gen Intern Med. 2017. doi:10.1007/s11606-016-3971-0 